\title{
Towards feedback stabilization over fading channels
}

\author{
(Invited Paper) \\ Paolo Minero, Massimo Franceschetti \\ Advanced Network Science \\ University of California \\ San Diego, CA, USA \\ Email: \{minero,massimo\}@ucsd.edu \\ Subhrakanti Dey, Girish Nair \\ Dept. of Electrical Engineering \\ University of Melbourne \\ Parkville Victoria 3010 Australia \\ Email: \{sdey,gnair\}@ee.unimelb.edu.au
}

\begin{abstract}
Motivated by control problems over wireless fading channels, the mean square stabilization of a linear system over a time-varying finite rate feedback link is studied. Process and observation disturbances are allowed to occur over an unbounded support. Necessary and sufficient conditions to stabilize the system are derived, and their implications are discussed. Proof techniques rely on both information theoretic and control theoretic tools.
\end{abstract}

\section{INTRODUCTION}

In wireless communication, the quality of the communication link between transmitter and receiver varies over time because of random fading in the received signal. In the case of digital communication, this can reflect in a time variation of the rate supported by the wireless channel. The coherence time indicates the time interval over which the channel can be considered constant. If the coherence time is long enough, then transmitter and receiver can estimate the quality of the link by sending a known sequence called pilot, and can adapt the communication scheme to the channel condition.

Let us assume the aim is to stabilize an unstable linear plant through a controller which receives measurements of a sensor over a fading wireless channel. Is it possible to design a communication scheme that changes dynamically according to the channel condition and, at the same time, is guaranteed to stabilize the system? This is the problem under investigation.

As a first approach, it is assumed that the communication channel is noiseless and, at any given time $k$, allows transmission of $R_{k}$ bits without error. The case when $R_{k}$ changes deterministically has been well studied. It has been shown, under different notions of stability and system models (see [5], [1], [3], [6] and references therein), that a discrete linear plant with parameter $|\lambda|>1$ can be stabilized if and only if the data rate $R_{k}$ over the digital link is no less than $\log _{2}|\lambda|$. This paper focuses on the case when $R_{k}$ fluctuates randomly. We model the coherence time by assuming that $R_{k}$ remains constant in blocks of $n$ consecutive channel uses and varies according to an independent and identically distributed (i.i.d) process across blocks. Channel state estimation at the transmitter and receiver is reinterpreted here as causal knowledge, at both encoder and decoder, of the rate supported by the communication link.

A work directly related to ours is [2]. These authors also considered the case of a random time-varying digital link and provided necessary and sufficient conditions for $m$-th moment stability. The main difference with our work is that we allow the system disturbance to have unbounded support, and the encoder has access to output feedback instead than state feedback. We point out that this is a more general model, which considerably complicates the design of the adaptive quantizer, as this must be capable of tracking the state when atypically large disturbances affect the system.

Some preliminary results are presented next. They consist of necessary and sufficient conditions on the channel rate to achieve second moment stability. A necessary condition to stabilize a linear system with parameter $|\lambda|>1$ in the second moment sense is,

$$
\mathbb{E}\left[\frac{|\lambda|^{2 n}}{2^{2 n R}}\right]<1,
$$

where $n$ is the length of the channel block with the same rate. Furthermore, it is shown that there exists a coder-decoder pair that stabilizes the system if,

$$
\mathbb{E}\left[\frac{|\lambda|^{2 n}}{2^{2 n R}}\right]<c,
$$

where $c \leq \frac{1}{16}$.

Condition (1) can be intuitively interpreted as follows. If no information is sent over the link during a transmission block, the estimation error at the controller grows by $|\lambda|^{n}$. The information sent by the coder can reduce this error by at most $2^{n R}$, where $n R$ is the total rate supported by the channel in a given block. However, if averaging over the fluctuation of the rate $|\lambda|^{n}$ exceeds $2^{n R}$, then the information rate of the channel cannot compensate (on average) the dynamics of the system and it is not possible to stabilize the plant. Notice that if the variation of the rate is deterministic, then our necessary condition reduces to the well known $R>\log _{2}|\lambda|$.

As for condition (2), we give an explicit construction of a coder-decoder pair similar to the one by Nair and Evans [1]. However, while in [1] the time variation of the transmission rate is part of the coder design, in the model under consideration the variation of $\left\{R_{k}\right\}_{k=1}^{\infty}$ is uniquely determined by a stochastic process. As the rate process is assumed constant over blocks of $n$ channels uses, coder and decoder exploit the causal knowledge of the rate, transmitting one packet per block, and by encoding each packet at the rate supported by the channel.

We notice that there is a constant gap between our necessary and sufficient conditions (1-2). We are currently working 
towards the possibility of closing this gap and we conjecture that (1) is indeed achievable using a more complex codingdecoding scheme than the one presented here. Although a suboptimal achievability result can be derived also in the case of multi-state discrete linear systems, in what follows for ease of exposition we limit the treatment only to the scalar case.

The rest of the paper is organized as follows. After introducing formally the stabilization problem, the necessity result is proved in Section III, while the sufficiency is developed in Section IV.

\section{PROBLEM FORMULATION}

In the sequel, expectation with respect to the random variable $X$ is written $\mathbb{E}_{X}[\cdot]$, the differential entropy of a continuous random variable $X$ as $h(X)=-\mathbb{E}\left[\ln f_{X}(x)\right]$, the entropy of a discrete random variable $X$ as $H(X)=$ $-\mathbb{E}[\ln P(X)]$ the set of non-negative integers as $\mathbb{W}$ and, finally, the positive integers as $\mathbb{Z}_{+}$.

Consider the scalar unstable stochastic linear system

$$
x_{k+1}=\lambda x_{k}+u_{k}+v_{k}, y_{k}=x_{k}+w_{k}, \forall k \in \mathbb{W}
$$

with state $x_{k}$, process disturbance $v_{k}$, control $u_{k}$, measurement $y_{k}$ and measurement noise $w_{k}$. It is assumed that $|\lambda|>1$, so that the system is unstable. No Gaussian assumptions are made on the disturbances, but the following assumptions are supposed to hold

A1. $x_{0}, v_{k}$ and $w_{j}$ are mutually independent for all $k, j \in \mathbb{W}$. A2. $\exists \epsilon>0$ such that $x_{0}, v_{k}$ and $w_{k}$ have uniformly bounded $(2+\epsilon)$ th absolute moments over $k \in \mathbb{W}$.

A3. $\inf _{k \in \mathbb{W}} h\left(v_{k}\right)>-\infty$. Thus, $\exists \beta>0$ such that $e^{2 h\left(v_{k}\right)}>$ $\beta$ for all $k \in \mathbb{W}$

Suppose that observer and controller are connected by a timevarying digital link. Suppose that the rate remains constant over blocks of $n \in \mathbb{Z}_{+}$channel uses but changes independently from block to block according to a given probability distribution. In each of the $n$ uses of the digital link during the $j$-th block symbols coming from an alphabet of size $2^{R_{j}}$ are transmitted without error from encoder to controller. Let $S_{k} \in\left\{1, \ldots, 2^{R_{j}}\right\}$ denote the symbol sent at time $k \in$ $\{j n, \ldots,(j+1) n-1\}$. An underlying stochastic process determines the alphabet size $2^{R_{j}}$ in each block. For simplicity, suppose that for all $j \in \mathbb{W} R_{j}$ are i.i.d random variables distributed as $R$, where $R$ can only take finite number of integer values, such that

$$
\mathbb{P}\left(R=r_{i}\right)=p_{i}, \quad i \in \mathcal{I} \subseteq \mathbb{Z}_{+},
$$

for $r_{i} \in \mathbb{W}, 0 \leq r_{\text {min }} \leq r_{i} \leq r_{\max }<\infty, \sum_{i \in \mathcal{I}} p_{i}=1$, and where $\mathcal{I}$ is a finite set. At the beginning of each block $j$, coder and decoder are assumed to know $R_{j}$ (and hence all $\left\{R_{i}\right\}_{i=0}^{j}$ ), while the realization of the rate process in future blocks, $\left\{R_{i}\right\}_{i=j+1}^{\infty}$, is unknown to them. The aim is to construct a coder-decoder which stabilizes the plant in the mean square sense

$$
\sup _{k \in \mathbb{W}} \mathbb{E}\left|x_{k}\right|^{2}<\infty
$$

using the finite data rate provided by the time-varying digital link. Each transmitted symbol can depend on all past and present measurements, the present channel state and the past symbols,

$$
\begin{aligned}
s_{k}= & g_{k}\left(y_{0}, \ldots, y_{k}, s_{0}, \ldots, s_{k-1}, R_{j}\right), \\
& k \in\{j n, \ldots,(j+1) n-1\}, \forall j \in \mathbb{W},
\end{aligned}
$$

where $g_{k}(\cdot)$ is the coder mapping at time $k$. The control sequence, on the other hand, can depend on all past and present channel symbols

$$
u_{k}=f_{k}\left(s_{0}, \ldots, s_{k}\right), \quad \forall k \in \mathbb{W},
$$

where $f_{k}(\cdot)$ is the controller mapping at time $k$. From (7),(6) and (3) observe that $x_{j n}$ does not depend on $R_{j}$ and furthermore $x_{j n} \rightarrow S_{0}^{j-1} \rightarrow R_{j}$ form a Markov chain.

\section{NECESSITY}

Theorem 3.1: Under the conditions above, necessary condition for stabilizability in mean square sense is that

$$
\mathbb{E}\left[\left(\frac{|\lambda|^{2}}{2^{2 R}}\right)^{n}\right]<1
$$

Proof: In order to prove the statement, we find a lower bound for the second moment of the state, and show that Eq. (8) is a necessary condition for the lower bound to be finite. We focus on the times $k=j n$ with $j \in \mathbb{W}$, i.e. the beginning of each channel block. Let $S_{0}^{j}=\left\{S_{0}, \ldots, S_{(j+1) n-1}\right\}$, denote the symbols sent over the noiseless channel until the end of the $j$-th channel block. By iteration of (3), we have

$x_{(j+1) n}=\lambda^{n} x_{j n}+\sum_{k=j n}^{(j+1) n-1} \lambda^{(j+1) n-1-k}\left[f_{k}\left(S_{0}, \ldots, S_{k}\right)+v_{k}\right]$

Let $n_{j}=\frac{1}{2 \pi e} \mathbb{E}_{S_{0}^{j-1}}\left[e^{2 h\left(x_{j n} \mid S_{0}^{j-1}=s_{0}^{j-1}\right)}\right]$ be the conditional entropy power of $x_{j n}$ conditioned on the event $\left\{S_{0}^{j-1}=\right.$ $\left.s_{0}^{j-1}\right\}$, averaged over all possible $s_{0}^{j-1}$. The second moment of $x_{j n}$ is lower bounded by $n_{j}$ :

$$
\begin{aligned}
\mathbb{E}\left[x_{j n}^{2}\right] & =\mathbb{E}_{S_{0}^{j-1}}\left[\mathbb{E}\left[x_{j n}^{2} \mid S_{0}^{j-1}=s_{0}^{j-1}\right]\right] \\
& =\frac{1}{2 \pi e} \mathbb{E}_{S_{0}^{j-1}}\left[e^{\ln \left(2 \pi e \mathbb{E}\left[x_{j n}^{2} \mid S_{0}^{j-1}=s_{0}^{j-1}\right]\right.}\right] \\
& \geq \frac{1}{2 \pi e} \mathbb{E}_{S_{0}^{j-1}}\left[e^{2 h\left(x_{j n} \mid S_{0}^{j-1}=s_{0}^{j-1}\right)}\right] \\
& =n_{j}
\end{aligned}
$$

where the inequality follows from the maximum entropy theorem [4, Theorem 9.4.1]. It follows that a necessary condition for (5) to hold is that $\sup _{j \in \mathbb{W}} n_{j}<\infty$. We will now complete the proof by showing that this necessary condition is violated whenever (8) does not hold. We make use of the following technical.

\section{Lemma 3.2:}

$\mathbb{E}_{S_{0}^{j} \mid S_{0}^{j-1}, R_{j}}\left[e^{2 h\left(x_{j n} \mid S_{0}^{j}=s_{0}^{j}\right)}\right] \geq \frac{1}{2^{2 n R_{j}}} e^{2 h\left(x_{j n} \mid S_{0}^{j-1}=s_{0}^{j-1}\right)}$ 
Proof: First, observe that the following chain of inequalities holds:

$$
\begin{aligned}
& \mathbb{E}_{S_{0}^{j} \mid S_{0}^{j-1}, R_{j}} h\left(x_{j n} \mid S_{0}^{j-1}=s_{0}^{j-1}, S_{0}^{j}=s_{0}^{j}, R_{j}\right) \\
& =h\left(x_{j n}, S_{0}^{j} \mid S_{0}^{j-1}=s_{0}^{j-1}, R_{j}\right)-H\left(S_{0}^{j} \mid S_{0}^{j-1}=s_{0}^{j-1}, R_{j}\right) \\
& \geq h\left(x_{j n} \mid S_{0}^{j-1}=s_{0}^{j-1}, R_{j}\right)-H\left(S_{0}^{j} \mid S_{0}^{j-1}=s_{0}^{j-1}, R_{j}\right) \\
& \geq h\left(x_{j n} \mid S_{0}^{j-1}=s_{0}^{j-1}, R_{j}\right)-\ln 2^{n R_{j}} \\
& =h\left(x_{j n} \mid S_{0}^{j-1}=s_{0}^{j-1}\right)-\ln 2^{n R_{j}}
\end{aligned}
$$

where $h(x, A \mid B)$ with $A$ discrete denotes $-\mathbb{E}\left[\ln \left(p_{A \mid B} f_{x \mid A, B}\right)\right]$. The last inequality follows from the fact that, given $R_{j}$, the cardinality of $\left\{S_{j n}, \ldots, S_{(j+1) n-1}\right\}$ is $2^{n R_{j}}$, and where the last equality follows from the fact that $x_{j n} \rightarrow S_{0}^{j-1} \rightarrow R_{j}$ is a Markov chain. Then,

$$
\begin{aligned}
& \mathbb{E}_{S_{0}^{j} \mid S_{0}^{j-1}, R_{j}} e^{2 h\left(x_{j n} \mid S_{0}^{j}=s_{0}^{j}\right)} \\
& \geq \mathbb{E}_{S_{0}^{j} \mid S_{0}^{j-1}, R_{j}} e^{2 h\left(x_{j n} \mid S_{0}^{j-1}=s_{0}^{j-1}, S_{0}^{j}=s_{0}^{j}, R_{j}\right)} \\
& \geq e^{2 \mathbb{E}_{S_{0}^{j} \mid S_{0}^{j-1}, R_{j}} h\left(x_{j n} \mid S_{0}^{j-1}=s_{0}^{j-1}, S_{0}^{j}=s_{0}^{j}, R_{j}\right)} \\
& \geq e^{2\left[h\left(X \mid S_{0}^{j-1}=s_{0}^{j-1}\right)-\ln 2^{n R_{j}}\right]} \\
& =\frac{1}{2^{2 n R_{j}}} e^{2 h\left(x_{j n} \mid S_{0}^{j-1}=s_{0}^{j-1}\right)}
\end{aligned}
$$

where the first inequality follows from the fact that conditioning reduces the entropy; the second inequality follows from Jensen's inequality; finally, (10) implies the third inequality.

Next, it is shown that $n_{j}$ evolves according to a recursive equation. Using standard properties of entropy [4] (translation invariance, conditional version of entropy power inequality), and assumptions A1. and A3., it follows that

$$
\begin{aligned}
& \mathbb{E}_{S_{0}^{j}}\left[e^{2 h\left(x_{(j+1) n} \mid S_{0}^{j}=s_{0}^{j}\right)}\right]= \\
& =\mathbb{E}_{S_{0}^{j}}\left[e^{2 h\left(\lambda^{n} x_{j n}+\sum_{k=j n}^{(j+1) n-1} \lambda^{(j+1) n-1-k} v_{k} \mid S_{0}^{j}=s_{0}^{j}\right)}\right] \\
& \geq \mathbb{E}_{S_{0}^{j}}\left[e^{2 h\left(\lambda^{n} x_{j n} \mid S_{0}^{j}=s_{0}^{j}\right)}+\sum_{k=j n}^{(j+1) n-1} e^{2 h\left(\lambda^{(j+1) n-1-k} v_{k} \mid S_{0}^{j}=s_{0}^{j}\right)}\right] \\
& =|\lambda|^{2 n} \mathbb{E}_{S_{0}^{j}}\left[e^{2 h\left(x_{j n} \mid S_{0}^{j}=s_{0}^{j}\right)}\right]+\sum_{k=j n}^{(j+1) n-1}|\lambda|^{2[(j+1) n-1-k]} e^{2 h\left(v_{k}\right)} \\
& \geq|\lambda|^{2 n} \mathbb{E}_{S_{0}^{j}}\left[e^{2 h\left(x_{j n} \mid S_{0}^{j}=s_{0}^{j}\right)}\right]+\sum_{k=j n}^{(j+1) n-1}|\lambda|^{2[(j+1) n-1-k]} \beta \\
& =|\lambda|^{2 n} \mathbb{E}_{S_{0}^{j}}\left[e^{2 h\left(x_{j n} \mid S_{0}^{j}=s_{0}^{j}\right)}\right]+\gamma \\
& =|\lambda|^{2 n} \mathbb{E}_{S_{0}^{j-1}, R_{j}}\left[\mathbb{E}_{S_{0}^{j} \mid S_{0}^{j-1}, R_{j}}\left[e^{2 h\left(x_{j n} \mid S_{0}^{j}=s_{0}^{j}\right)}\right]\right]+\gamma \\
& \geq|\lambda|^{2 n} \mathbb{E}_{S_{0}^{j-1}, R_{j}}\left[\frac{1}{\left|2^{n R_{j}}\right|^{2}} e^{2 h\left(x_{j n} \mid S_{0}^{j-1}=s_{0}^{j-1}\right)}\right]+\gamma \\
& =|\lambda|^{2 n} \mathbb{E}_{R_{j}}\left[\frac{1}{\left.\mid 2^{n R_{j}||^{2}}\right]} \mathbb{E}_{S_{0}^{j-1}}\left[e^{2 h\left(x_{j n} \mid S_{0}^{j-1}=s_{0}^{j-1}\right)}\right]+\gamma,\right.
\end{aligned}
$$

the second inequality uses assumption A3. above, i.e. $e^{\frac{2}{f} h\left(v_{k}\right)}>\beta$. The constant $\gamma$ is defined as and $\gamma:=$ $\sum_{k=j n}^{(j+1) n-1}|\lambda|^{2[(j+1) n-1-k]} \beta$. Finally, the last inequality follows from Lemma 3.2 and the fact that $R_{j}$ is independent of $x_{j n}$ and $S_{0}^{j-1}$. Thus, using the fact that the rate process is i.i.d.,

$$
n_{j+1} \geq \mathbb{E}\left[\frac{|\lambda|^{2 n}}{2^{2 n R}}\right] n_{j}+\frac{\gamma}{2 \pi e} .
$$

Therefore, $\mathbb{E}\left[\frac{|\lambda|^{2 n}}{2^{2 n R}}\right] \geq 1$ implies that $\sup _{j \in \mathbb{W}} n_{j}=\infty$.

\section{SUFFICIENCY}

Proposition 4.1: Suppose that $n r_{\min } \geq 2$. Under the conditions above, sufficient condition for stabilizability in mean square sense is that

$$
\mathbb{E}\left[\left(\frac{|\lambda|^{2}}{2^{2 R}}\right)^{n}\right]<\frac{1}{\phi \zeta}
$$

where $\phi$ and $\zeta$ are constants such that $\phi=2^{1+\epsilon} \geq 2$ and $\zeta \geq 8$.

The remaining part of the paper is dedicated to the proof of the above Proposition. The coder/decoder pair is based on the construction given in [1]. The coder utilizes the quantizer that is described below.

\section{A. Quantizer}

The quantizer partitions the real line into non-uniform regions, in such a way that most of the regions are concentrated in a symmetrical way around the origin. A parameter $\rho>1$ determines the speed at which the quantizer range increases. The quantizer generates $2^{\nu}, \nu \geq 2$, quantization intervals by partitioning the set $[-1,1]$ into $2^{\nu-1}$ intervals of equal length, and the sets $\left(\rho^{i-2}, \rho^{i-1}\right],\left[-\rho^{i-2},-\rho^{i-1}\right)$ into $2^{\nu-i}$ intervals of equal length, $i \in[2, \ldots, \nu]$. The two open sets $\left(\rho^{\nu-1}, \infty\right)$ and $\left(-\infty,-\rho^{\nu-1}\right]$ are respectively the leftmost and rightmost intervals of the quantizer. Note that as $i$ increases the size of the intervals increases. We assume that $I(0), \ldots, I\left(2^{\nu}-1\right)$ are labels for the intervals from left to right. Let

- $\kappa_{\nu}(\omega)$ be half-length of interval $I(\omega)$ for $\omega \in$ $\left[1, \ldots, 2^{n-2}\right]$, be equal to $\rho^{\nu}-\rho^{\nu-1}$ when $\omega=2^{\nu}-1$ and equal to $-\left(\rho^{\nu}-\rho^{\nu-1}\right)$ when $\omega=0$.

- $q_{\nu}(x):=\bar{\omega}_{\nu}(\omega)$ be midpoint of interval $x \in I(\omega)$ for $\omega \in\left[1, \ldots, 2^{\nu-2}\right]$, be equal to $\rho^{\nu}$ when $\omega=2^{\nu}-1$ and equal to $-\rho^{\nu}$ when $\omega=0$.

If a real number $x$ is in $I(\omega)$ for some $\omega \in\left\{0, \ldots, 2^{\nu-1}\right\}$, then the quantizer approximates $x$ with $\bar{\omega}_{\nu}(\omega)$. The quantization error is not uniform over $x \in \mathbb{R}$, but is bounded by $\kappa_{\nu}(\omega)$ for all $\omega \in\left\{1, \ldots, 2^{\nu-2}\right\}$. Suppose now that $X$ is a real-valued random variable. A fundamental property of the quantizer is that the average quantization error diminish like the inverse square of the number of levels, $2^{-\nu}$. More precisely, if the $(2+\epsilon)$-th moment of $X$ is bounded for some $\epsilon>0$, then an upper bound of the second moment of the estimation error decays as $2^{-\nu}$. The higher moment of $X$ is useful to bound the estimation error (using Chebyshev's inequality) when $X$ lies in one of the two open intervals $\left(\rho^{\nu-1}, \infty\right)$ and $\left(-\infty,-\rho^{\nu-1}\right]$. 
Let $L$ be a strictly positive random variable, define the functional

$$
M_{\epsilon}[X, L] \equiv \mathbb{E}\left[L^{2}+|X|^{2+\epsilon} L^{-\epsilon}\right] .
$$

The functional $M_{\epsilon}[X, L]$ is an upper bound to the second moment of $X$ :

$$
\mathbb{E}\left[|X|^{2}\right]=\mathbb{E}\left[|X|^{2}\left(1_{|X| \leq L}+1_{|X|>L}\right)\right] \leq M_{\epsilon}\{X \mid L\}
$$

Given a third random variable $R$, define the conditional version of $M_{\epsilon}[X, L]$ given $R$ as

$$
M_{\epsilon}[X, L \mid R] \equiv \mathbb{E}\left[L^{2}+|X|^{2+\epsilon} L^{-\epsilon} \mid R\right]
$$

The fundamental property of the quantizer described above is given by the following result:

Lemma 4.2: Let $X$ and $L>0$ and $R \geq 2 / n$ be random variables with $\mathbb{E} X^{2+\epsilon}<\infty$ for some $\epsilon>0$, and $n \in \mathbb{W}$. If $\rho>2^{2 / \epsilon}$, then the quantization error $X-L q_{n R}(X / L)$ satisfies

$$
M_{\epsilon}\left[X-L q_{\nu}(X / L), L \kappa_{n R}(\omega) \mid R\right] \leq \frac{\zeta}{2^{2 n R}} M_{\epsilon}[X, L]
$$

for some $\zeta \geq 8$ determined only by $\epsilon$ and $\rho$.

Proof: [1, Lemma 5.2]

Next, the coder and observer are described.

\section{B. Coder}

The first stage of the encoding process consists of computing the linear minimum variance estimator of the plant state based on the previous measurements and control sequences. The filter process satisfies a recursive equation of the same form as (3), namely

$$
\bar{x}_{k+1}=\lambda \bar{x}_{k}+u_{k}+z_{k}, z \in \mathbb{W} .
$$

where the innovation $z_{k}$ is uniformly bounded. From the orthogonality principle the stability of $\bar{x}$ is equivalent to that of $x$. The output $\bar{x}_{k}$ of the filter (or a function of it) must be transmitted using the finite number of bits supported on the digital channel. Coder and decoder share a state estimator $\hat{x}_{k}$ based uniquely on the symbols sent on the digital link. Since $\hat{x}_{k}$ is available both at the coder and decoder, while the minimum variance estimator is available at the coder only, the encoder utilizes a predictive quantizer to encode the error between $\bar{x}_{k}$ and $\hat{x}_{k}$. The error is scaled by an appropriate coefficient and then quantized using the quantizer in Section IV-A. Just before the start of the $j$-th block at time $k=j n$ the coder sets the quantization rate $\nu$ equal to $n R_{j}$ and computes

$$
\bar{\omega}_{n R_{j}}\left(\omega_{j}\right)=q_{n R_{j}}\left(\frac{\bar{x}_{j n}-\hat{x}_{j n}}{l_{j}}\right)
$$

where $l_{j}$ is a scaling factor updated after each packet transmission. This factor is utilized to scale $\bar{x}_{j n}-\hat{x}_{j n}$ close to the origin, where the quantizer provides better estimates. The index $\omega_{j}$ of the quantization level is converted into a string of $n R_{j}$ bits and transmitted using the $n$ channel uses of the $j$-th block.

Before the $(j+1)$-th block, the coder updates the state estimator as follows

$$
\begin{aligned}
\hat{x}_{(j+1) n} & =\lambda^{n}\left[\hat{x}_{j n}+l_{j} \bar{\omega}_{n R}\left(\omega_{j}\right)\right]+ \\
& +\sum_{k=j n}^{(j+1) n-1} \lambda^{(j+1) n-1-k} L \hat{x}_{k}
\end{aligned}
$$

where

$$
\hat{x}_{k+1}=(\lambda+L) \hat{x}_{k}, \quad \forall k \in[j n, \ldots, j n+n-2]
$$

and $\hat{x}_{0}=0 . L$ is the certainty-equivalent control coefficient. Finally the scaling coefficient $l_{j}$ is updated as follows

$$
l_{j+1}=\max \left\{\sigma, l_{j}|\lambda|^{n} \kappa_{n R_{j}}\left(\omega_{j}\right)\right\}
$$

with $l_{0}=\sigma$, where $\sigma^{2+\epsilon}$ is a uniform bound in the $(2+\epsilon)$ moment of

$$
g_{j}:=\sum_{i=0}^{n-1} \lambda^{n-i} z_{j n+i}, \quad j \in \mathbb{W}
$$

It is important to note from (20) and the independence of the rate process that the $l_{j}$ does not depend on $R_{j}$.

\section{Controller}

At time $k=j n$ coder and controller are synchronized and have common knowledge of the state estimator $\hat{x}_{j n}$. While receiving the digitalized index of (17) during times $j n, \ldots, j n+n-2$, the controller sends to the plant a certaintyequivalent control signal

$$
u_{k}=L \hat{x}_{k} \quad \forall k \in[j n, \ldots, j n+n-2]
$$

where $\hat{x}_{k}$ is updated as in (19). Once received $\bar{\omega}_{n R}\left(\omega_{j}\right)$, the controller updates the estimator $\hat{x}_{(j+1) n}$ using (18). Synchronism between coder and observer is ensured by the fact that the initial value $\hat{x}_{0}$ is set equal to zero at both coder and decoder, and by the fact that the digital link is noiseless.

\section{Analysis}

In this section it is shown that the coder/controller pair described above ensures that the second moment of $\bar{x}$ is uniformly bounded if (11) is satisfied. Define the coder error at time $k \in \mathbb{W}$ as $f_{k}=\hat{x}_{k}-\bar{x}_{k}$.

The following analysis is developed in three steps. First it is shown that $f_{k}$ is bounded for all times $k=j n j \in \mathbb{W}$, i.e. the beginning of each channel block. Next, the analysis is extended to all $k \in \mathbb{W}$. Finally the stability of $f_{k}$ for all $k \in \mathbb{W}$ is shown to imply that $\bar{x}$ (and so $x$ ) is uniformly bounded.

First we show that the coder error $f_{k}=\hat{x}_{k}-\bar{x}_{k}$ is bounded in mean square for all times $k=j n j \in \mathbb{W}$. Instead of looking at directly at $\mathbb{E}\left[f_{j n}^{2}\right]$, it is more convenient to consider the functional $M_{\epsilon}[X, L]$ defined in (12), with $X=f_{j n}$ and $L=$ $l_{j}$. Thus, let

$$
\theta_{j}:=M_{\epsilon}\left\{f_{j n}, l_{j}\right\} \equiv \mathbb{E}\left[l_{j}^{2}+\left|f_{j n}\right|^{2+\epsilon} l_{j}^{-\epsilon}\right]
$$

Equation (13) implies that $\mathbb{E}\left[f_{j n}^{2}\right]<\theta_{j}$. Therefore, it suffices to show that $\sup _{j \in \mathbb{W}} \theta_{j}<\infty$. 
Substituting (22) into (16), and iterating over a block It follows that duration

$$
\begin{aligned}
\bar{x}_{(j+1) n} & =\lambda^{n} \bar{x}_{j n}+\sum_{k=j n}^{(j+1) n-1} \lambda^{(j+1) n-1-k}\left(L \hat{x}_{k}+z_{k}\right) \\
& =\lambda^{n} \bar{x}_{j n}+g_{j}+\sum_{k=j n}^{(j+1) n-1} \lambda^{(j+1) n-1-k}\left(L \hat{x}_{k}\right)
\end{aligned}
$$

where $g_{j}$ is defined in (21). So subtracting (23) from (18)

$$
\begin{aligned}
f_{(j+1) n} & =\hat{x}_{(j+1) n}-\bar{x}_{(j+1) n} \\
& =\lambda^{n}\left[f_{j n}-l_{j} \bar{\omega}_{n R_{j}}\left(\omega_{j}\right)\right]+g_{j} .
\end{aligned}
$$

Next, $f_{(j+1) n}$ is used to derive an expression for $\theta_{j+1}$. From the inequality $(|x|+|y|)^{\alpha} \leq 2^{\alpha-1}\left(|x|^{\alpha}+|y|^{\alpha}\right) \forall \alpha>0$,

$\left|f_{(j+1) n}\right|^{2+\epsilon} \leq \phi\left(\left|\lambda^{n}\right|^{2+\epsilon}\left|f_{j n}-l_{j} q_{n R_{j}}\left(f_{j n} / l_{j}\right)\right|^{2+\epsilon}+\left|z_{j}\right|^{2+\epsilon}\right)$ with $\phi=2^{1+\epsilon}$. Dividing by $l_{j+1}^{\epsilon}$ and taking expectations,

$$
\begin{aligned}
& \mathbb{E}\left[\left|f_{(j+1) n}\right|^{2+\epsilon} l_{j+1}^{-\epsilon}\right] \\
& \leq \phi\left(\left|\lambda^{n}\right|^{2+\epsilon} \mathbb{E}\left[\frac{\left|f_{j n}-l_{j} q_{n R_{j}}\left(f_{j n} / l_{j}\right)\right|^{2+\epsilon}}{l_{j+1}^{\epsilon}}\right]+\mathbb{E}\left[\frac{\left|g_{j}\right|^{2+\epsilon}}{l_{j+1}^{\epsilon}}\right]\right) \\
& \leq \phi\left(\left|\lambda^{n}\right|^{2+\epsilon} \mathbb{E}\left[\frac{\left|f_{j n}-l_{j} q_{n R_{j}}\left(f_{j n} / l_{j}\right)\right|^{2+\epsilon}}{\left[l_{j}\left|\lambda^{n}\right| \kappa_{n R_{j}}\left(\omega_{j}\right)\right]^{\epsilon}}\right]+\mathbb{E}\left[\frac{\left|g_{j}\right|^{2+\epsilon}}{\sigma^{\epsilon}}\right]\right) \\
& =\phi\left(\left|\lambda^{n}\right|^{2} \mathbb{E}\left[\frac{\left|f_{j n}-l_{j} q_{n R_{j}}\left(f_{j n} / l_{j}\right)\right|^{2+\epsilon}}{\left[l_{j} \kappa_{n R_{j}}\left(\omega_{j}\right)\right]^{\epsilon}}\right]+\mathbb{E}\left[\frac{\left|g_{j}\right|^{2+\epsilon}}{\sigma^{\epsilon}}\right]\right),
\end{aligned}
$$

where we used the fact that, from (20), $l_{j+1}=$ $\max \left\{\sigma, l_{j}\left|\lambda^{n}\right| \kappa_{n R_{j}}\left(\omega_{j}\right)\right\}$. Observe that

$$
\begin{aligned}
\mathbb{E}\left[l_{j+1}^{2}\right] & \equiv \mathbb{E}\left[\max \left\{\sigma^{2}, l_{j}^{2}\left|\lambda^{n}\right|^{2}\left[\kappa_{n R_{j}}\left(\omega_{j}\right)\right]^{2}\right\}\right] \\
& \leq \sigma^{2}+\left|\lambda^{n}\right|^{2} \mathbb{E}\left[\left|l_{j} \kappa_{n R_{j}}\left(\omega_{j}\right)\right|^{2}\right] .
\end{aligned}
$$

Adding this to (25), using $\mathbb{E}\left[g_{j}^{2+\epsilon}\right]<\sigma^{2+\epsilon}$ and the definition of $\theta_{j}$,

$$
\begin{aligned}
\theta_{j+1} \leq & \phi\left(2 \sigma^{2}+\right. \\
& \left.\left|\lambda^{n}\right|^{2} \mathbb{E}\left\{\frac{\left|f_{j n}-l_{j} q_{n R_{j}}\left(f_{j n} / l_{j}\right)\right|^{2+\epsilon}}{\left[l_{j} \kappa_{n R_{j}}\left(\omega_{k}\right)\right]^{\epsilon}}+\left|l_{j} \kappa_{n R_{j}}\left(\omega_{j}\right)\right|^{2}\right\}\right) \\
= & \phi\left(2 \sigma^{2}+\right. \\
& \left.\left|\lambda^{n}\right|^{2} \mathbb{E}_{R_{j}}\left[M_{\epsilon}\left\{f_{j n}-l_{j} q_{n R_{j}}\left(\frac{f_{j n}}{l_{j}}\right), l_{j} \kappa_{n R_{j}}\left(\omega_{j}\right) \mid R_{j}\right\}\right]\right) \\
\leq & \phi\left(2 \sigma^{2}+\left|\lambda^{n}\right|^{2} \mathbb{E}_{R_{j}}\left[\frac{\zeta}{2^{2 n R_{j}}} M_{\epsilon}\left\{f_{j n} \mid l_{j}\right\}\right]\right) \\
= & \phi 2 \sigma^{2}+\phi \zeta \mathbb{E}\left[\frac{\left|\lambda^{n}\right|^{2}}{2^{2 n R}}\right] \theta_{j},
\end{aligned}
$$

where the second inequality follows from Lemma (4.2), and the last equality uses the fact that the rate process is i.i.d. and that $f_{j n}$ and $l_{j}$ are independent of $R_{j}$. Therefore, $\theta_{j}$ evolves according to the following recursive equation

$$
\theta_{j+1}=\phi 2 \sigma^{2}+\phi \zeta \mathbb{E}\left[\frac{\left|\lambda^{n}\right|^{2}}{2^{2 n R}}\right] \theta_{j}
$$

$$
\mathbb{E}\left[\frac{\left|\lambda^{n}\right|^{2}}{2^{2 n R}}\right]<\frac{1}{\phi \zeta},
$$

where $\phi=2^{1+\epsilon}$ and $\zeta \geq 8$, is a sufficient condition to have $\sup _{j \in \mathbb{W}} \theta_{j}<\infty$. Hence, from (13) it follows that $\mathbb{E}\left[f_{j n}\right]^{2}$ is bounded for every $j \in \mathbb{W}$.

Next, for any $r \in\{0, n-1\}$ triangle inequality implies

$$
\left|f_{j n+r}\right| \leq|\lambda|^{r}\left|f_{j n}\right|+\sum_{k=0}^{r-1}\left|\lambda^{r-1-k} L\right|\left|z_{j n+k}\right|,
$$

so the error $f_{k}$ is uniformely bounded for all $k \in \mathbb{W}$.

Finally, by rewriting (16) as

$$
\bar{x}_{k+1}=(\lambda+L) \bar{x}_{k}-L f_{k}+z_{k},
$$

the fact that $f_{k}$ and $z_{k}$ are uniformly bounded and the strict 'stability of $\lambda+L$ ensures that $\mathbb{E} \bar{x}_{k}^{2}<\infty$ for all $k \in \mathbb{W}$.

\section{CONCLUSION}

Motivated by control problems over wireless fading channels, we considered mean square stabilizability of a discretetime, linear system with a noiseless time-varying digital communication link. Process and observation disturbances are allowed to occur over an unbounded support. Necessary and a sufficient conditions to stabilize the system are derived.

\section{ACKNOWLEDGMENT}

This work is partially supported by the National Science Foundation CAREER award CNS-0546235 and NSF grant CCF 0635048.

\section{REFERENCES}

[1] G. N. Nair and R. J. Evans, "Stabilizability of stochastic linear systems with finite feedback data rates", SIAM Journal on Control and Optimization, Society for Industrial and Applied Mathematics, USA, vol. 43, no. 2, pp. 413 - 436, July 2, 2004.

[2] N.C. Martins, M.A. Dahleh and N.Elia, "Feedback stabilization of uncertain systems in the presence of a direct link", IEEE Transactions on Automatic Control, Volume 51, Issue 3, March 2006 Page(s):438 - 447.

[3] S. Tatikonda and S. Mitter "Control under communication constraints", Automatic Control, IEEE Transactions on, Volume 49, Issue 7, July 2004 Page(s): 1056 - 1068

[4] T. Cover and J. Thomas, Elements of Information Theory. New York: Wiley, 1987.

[5] W.S. Wong and R.W. Brockett, "Systems with finite communication bandwidth constraints. II. Stabilization with limited information feedback", IEEE Transactions on Automatic Control, Volume 44, Issue 5, May 1999

[6] A. Sahai and S. Mitter "The Necessity and Sufficiency of Anytime Capacity for Stabilization of a Linear System Over a Noisy Communication LinkPart I: Scalar Systems", IEEE Transactions on Information Theory, Volume 52, Issue 8, Aug. 2006 